\title{
Mobilidade funcional, força, medo de cair, estilo e qualidade de vida em idosos praticantes de caminhada
}

\section{Functional mobility, strength, fear of falling, lifestyle and quality of life in elderly practitioners of walking}

Clévia da Silva ${ }^{1}$, Natália Cristina de Oliveira ${ }^{2}$, (DFábio Marcon Alfieri ${ }^{2}$

\section{RESUMO}

O processo de envelhecimento envolve modificações no desempenho locomotor que podem predispor os idosos a quedas e alterações na qualidade de vida. Objetivo: Comparar o medo de cair, mobilidade funcional, força de membros inferiores, estilo e qualidade de vida em idosos sedentários e praticantes caminhada. Método: Trata-se de estudo transversal observacional do qual participaram 51 idosos, 25 praticantes de caminhada (GCam) e 26 sedentários (GSed), submetidos à avaliação de mobilidade funcional (Timed Up and Go - TUG), da força de membros inferiores (teste senta-levanta), medo de cair (Escala Internacional de Eficácia de Quedas - FES-I), estilo de vida (questionário FANTASTICO) e Qualidade de vida (SF-36). Resultados: Não houve diferença entre os grupos em relação às médias de idade, distribuição de gênero e índice de massa corporal. Os participantes do GCam apresentaram mobilidade funcional significantemente melhor $(9,45 \pm 2,68$ vs. $14,97 \pm 6,55$ segundos; $p=0,001)$ que os do GSed, e menos medo de cair $(23,16 \pm 5,33$ vs. $29,04 \pm 10,22 ; p=0,01)$. O estilo de vida também foi superior entre os praticantes de caminhada $(79,84 \pm 5,52$ vs. $67,19 \pm 10,35 ;(p=0,0001)$. Em relação à qualidade de vida, o GCam apresentou escores maiores nos domínios capacidade funcional $(p=0,013)$, limitações por aspecto físico $(p=0,17)$ e limitações por aspectos emocionais $(p=0,05)$. Já no domínio dor, o GCam apresentou pior resultado $(p=0,05)$ em relação ao GSed. Conclusão: Idosos praticantes regulares de caminhada possuem melhor resultados em relação à mobilidade funcional, habilidade de levantar e sentar, menos medo de cair, melhor estilo de vida e alguns domínios da qualidade de vida do que idosos sedentários.

Palavras-chave: Idoso, Limitação da Mobilidade, Aptidão Física, Estilo de Vida, Qualidade de Vida

${ }^{1}$ Discente, Mestrado em Promoção da Saúde, Centro Universitário Adventista de São Paulo - UNASP.

${ }^{2}$ Docente, Mestrado em Promoção da Saúde, Centro Universitário Adventista de São Paulo - UNASP.

\section{Correspondência}

Fábio Marcon Alfieri

E-mail: fabio.alfieri@unasp.edu.br

Recebido: 23 Março 2018.

Aceito: 20 Junho 2018.

\section{Como citar}

Silva C, Oliveira NC, Alfieri FM. Mobilidade funcional, força, medo de cair, estilo e qualidade de vida em idosos praticantes de caminhada.

Acta Fisiatr. 2018;25(1):22-26.

\begin{abstract}
The aging process involves changes in locomotor performance and may predispose the elderly to falls and changes in quality of life. Objective: Compare the fear of falling, functional mobility, lower limbs strength, lifestyle and quality of life in sedentary elderly and walking practitioners. Method: It is an observational cross-sectional study involving 51 elderly persons, 25 regular walkers (RW) and 26 sedentary (ST) participants. Participants were evaluated for functional mobility (Timed Up and Go - TUG), lower limb strength (stand and sit test), fear of falling (Falls Efficacy Scale - International - FES-I), lifestyle (FANTASTICO questionnaire) and quality of life (SF-36). Results: There were no differences between both groups regarding mean age, gender distribution and body mass index. Participants from RW displayed significantly better functional mobility $(9.45 \pm 2.68$ vs. $14.97 \pm 6.55$ seconds, $p=0.001)$ than those from ST, as well as less fear of falling $(23.16 \pm 5.33$ vs. $29.04 \pm 10.22$, $p=0.01)$. Lifestyle was also higher among walking practitioners (79.84 \pm 5.52 vs. $67.19 \pm 10.35$, $p=0.0001)$. Regarding quality of life, RW presented higher scores in functional capacity $(p=0.013)$, functional limitations $(p=0.17)$, and limitations due to emotional aspects $(p=0.05)$ when compared to the ones in SG. Conclusion: Elderly who practice regular walking have better results regarding functional mobility, ability stand and sit down, less fear of falling, better lifestyle and better quality of life in some domains when compared to sedentary elderly subjects.
\end{abstract}

Keywords: Aged, Mobility Limitation, Physical Fitness, Life Style, Quality of Life 


\section{INTRODUÇÃO}

O envelhecimento populacional é um fenômeno que vem aumentando significativamente no Brasil e no mundo. ${ }^{1}$ Durante este período da vida, diversas modificações acontecem na vida dos idosos, como alterações fisiológicas que alteram o desempenho locomotor e que podem predispor estes indivíduos a quedas e alterações na qualidade de vida. ${ }^{2-4}$

Devido a estas alterações, são necessárias intervenções por meio de atividades físicas, que podem interferir positivamente nas capacidades físicas e na qualidade de vida destes indivíduos. ${ }^{5}$

Sabe-se que a prática regular de atividades físicas proporciona diversos benefícios à saúde de idosos. Desde o aumento da expectativa de vida6, controle e diminuição de riscos de doenças cardiovasculares, ${ }^{7}$ ósseas, ${ }^{8}$ mentais, ${ }^{9}$ e pode promover até mesmo melhoria no bem-estar. ${ }^{10} \mathrm{~A}$ prática regular de atividade física pode promover aumento da capacidade aeróbia, força muscular, capacidade cognitiva, dentre outras capacidades e funções que tornam este tipo de intervenção essencial durante o envelhecimento. ${ }^{11,12}$

Em que pesem todos os benefícios, o sedentarismo é um fenômeno global e que afeta também os indivíduos idosos. ${ }^{13}$ Portanto, o incentivo para a prática de qualquer tipo de atividade física regular nessa faixa etária deve ser incentivada. ${ }^{14}$

A caminhada é uma das atividades físicas recomendadas para indivíduos idosos pelo fato de ser um exercício de baixo impacto, que envolve grandes grupos musculares, que pode contribuir para o envolvimento social, e que resulta em benefícios para a saúde. ${ }^{15}$ Ainda, a caminhada, não requer habilidades ou condições especiais e é viável para praticamente todas as faixas etárias já que envolve baixo risco de lesões. ${ }^{16}$

Por se tratar de uma atividade de fácil acesso, baixo custo e com poucas contraindicações, a caminhada é uma atividade física comum entre idosos. Sabe-se que esta prática tem um papel importante na prevenção primária e secundária de doenças cardiovasculares por exemplo, ${ }^{17}$ e é considerada um excelente tipo de exercício. ${ }^{18}$

Apesar disso, os diversos benefícios da caminhada para indivíduos idosos ainda não foram completamente explorados pela literatura. Assim, estudos sobre os efeitos da caminhada em aspectos relacionados às capacidades motoras como a força muscular e mobilidade funcional se tornam desejáveis a fim de se conhecer os reais efeitos desta prática comum sobre a vida de indivíduos idosos.

\section{OBJETIVO}

O objetivo deste estudo foi comparar - medo de cair, a mobilidade funcional, habilidade de levantar e sentar, estilo e qualidade de vida em indivíduos idosos sedentários e praticantes de caminhada.

\section{MÉTODO}

Este estudo teve caráter transversal observacional e seus procedimentos foram aprovados pelo comitê de ética do Centro Universitário Adventista de São Paulo (parecer número 1.553.237). Todos os participantes do estudo assinaram um termo de consentimento livre e esclarecido.

A amostra foi composta por 51 idosos (idade igual ou superior a 60 anos) de ambos os sexos, selecionados por conveniência dentre os pacientes cadastrados em uma Unidade Básica de Saúde (UBS) da região Sul da Cidade de São Paulo (distrito Capão Redondo).

Foram excluídos do estudo idosos acamados, que possuíssem limitação física ou comprometimento hemodinâmico que impedisse a realização de caminhada, aqueles com próteses nos membros inferiores, osteossínteses em membros inferiores, diabetes e hipertensão não controladas, alterações cognitivas, déficit visuais não corrigidos, com queixa de tontura, ou que apresentassem artropatias nas articulações com intensidade de dor igual ou superior a 4 $\mathrm{cm}$ na Escala Visual Analógica. ${ }^{19}$

Vinte e cinco dos 51 voluntários eram participantes regulares do programa de caminhada mantido pela UBS. O programa funciona regularmente há quatro anos, sendo realizado com frequência de duas vezes por semana, com duração de uma hora por sessão. Os indivíduos do Grupo Caminhada (GCam) frequentavam as atividades do grupo há pelo menos seis meses com presença em no mínimo $75 \%$ das sessões de caminhada por mês.

O programa de caminhada tem como objetivo estimular a atividade física e melhorar a qualidade e estilo de vida dos usuários através da prevenção de danos causados pelo sedentarismo. O grupo é acompanhado por fisioterapeutas e demais componentes da Equipe de Saúde da Família.
O grupo de idosos sedentários (GSed, $\mathrm{n}=26$ ) foi selecionado aleatoriamente a partir de uma lista contendo todos os indivíduos cadastrados na UBS. Além de passarem pelos mesmos critérios de inclusão, os participantes do GSed não participavam de atividades físicas regulares (duas ou mais vezes por semana) de qualquer natureza, há pelo menos 6 meses.

Todos os participantes da pesquisa foram inicialmente submetidos a uma avaliação inicial composta por testes de mobilidade funcional, força de membros inferiores, medo de quedas, estilo de qualidade de vida.

A mobilidade funcional foi avaliada pelo teste Timed Up and Go. Este teste avalia o nível de mobilidade individual, mensurando em segundos o tempo gasto pelo voluntário para levantar-se de uma cadeira (sem ajuda dos braços), percorrer uma distância de 3 metros, e retornar e sentar-se novamente. 0 teste foi realizado uma vez para familiarização e uma segunda vez para a tomada do tempo. ${ }^{20}$

Para verificar a força de membros inferiores aplicou-se o teste senta-levanta da cadeira que avalia força e equilíbrio. $O$ teste foi realizado com auxílio de uma cadeira sem braço onde o participante sentava-se com a coluna ereta, pés separados numa distância equivalente à largura dos ombros e braços cruzados sobre o tórax. Foi solicitado a cada voluntário, levantar-se e sentar-se cinco vezes consecutivas o mais rápido possível. O tempo gasto para realizar esta tarefa foi cronometrado. ${ }^{21-23}$

Para avaliar a preocupação a respeito da possibilidade de cair foi aplicada a Escala Internacional de Eficácia de Quedas (FES-I). Esta escala avalia o medo de queda durante a execução de 16 atividades cotidianas. A pontuação varia de 1 ("não estou preocupado") a 4 ("muito preocupado"). O escore total pode variar entre 16 e 64 pontos, no qual o menor valor corresponde à ausência de preocupação e quanto maior o valor, maior a preocupação em cair. ${ }^{24}$

Para conhecer a qualidade de vida foi aplicado o questionário Medical Outcomes Study 36-item Short-Form Health Survey (SF-36). ${ }^{25}$ Os itens são agrupados em oito domínios: capacidade funcional, aspectos físicos, dor, estado geral de saúde, vitalidade, aspectos sociais, aspectos emocionais, saúde mental e uma questão de avaliação comparativa entre as condições de saúde atual e de um ano atrás. $O$ instrumento analisa tanto aspectos negativos (doença ou enfermidade), como positivos (bem-estar). Os dados foram avaliados a partir da transformação das respostas em escores em uma escala de 0 a 
100 para cada componente, não havendo um único valor que resuma toda a avaliação.

Para avaliar o estilo de vida foi usada a versão validada em português do questionário "Estilo de vida FANTASTICO", um instrumento genérico que considera o comportamento dos indivíduos no último mês. ${ }^{26} \mathrm{O}$ instrumento é composto por 25 questões divididas em 9 domínios: família e amigos; atividade física; nutrição; cigarro e drogas; álcool; sono, cinto de segurança, estresse e sexo seguro; tipo de comportamento; introspecção e trabalho. As possíveis respostas são organizadas em escala Likert, e a soma dos pontos fornece um escore que classifica o estilo de vida dos indivíduos em excelente (85-100 pontos), muito bom (70-84 pontos), bom (55-69 pontos), regular (35-54 pontos, e necessita melhorar (0-34 pontos).

A análise dos dados foi realizada pelo pacote estatístico Graph Pad Instat. Os dados estão apresentados em médias \pm desviospadrão. As comparações entre os grupos foram feitas através do teste t e teste exato de Fisher. Em todos os casos, o nível descritivo $\alpha$ estabelecido foi de $5 \%(p<0,05)$.

\section{RESULTADOS}

Participaram deste estudo 51 indivíduos de ambos os sexos com idade variando entre 60 e 80 anos. Os participantes do GCam e GSed eram semelhantes em relação às médias de idade, peso corporal, estatura, índice de massa corporal (IMC) e distribuição de gêneros (Tabela 1).
Ao comparar os resultados dos testes clínicos e do estilo de vida entre os grupos, observou-se que em todos eles, os participantes do GCam obtiveram resultados melhores do que os indivíduos do GSed (Tabela 2).

Em relação à qualidade de vida, o escore geral não revelou diferença entre os grupos. Entretanto, ao avaliar os domínios do instrumento, o GCam apresentou escores estatisticamente superiores no que diz respeito à capacidade funcional, limitação por aspectos físicos e limitação por aspectos emocionais, e escore estatisticamente mais baixo no domínio dor (Tabela 3).

\section{DISCUSSÃO}

O objetivo deste estudo foi comparar o medo de cair, a mobilidade funcional, habilidade de levantar e sentar, estilo e qualidade de vida em indivíduos idosos sedentários e praticantes de caminhada. Os resultados mostraram que indivíduos que praticam caminhada regularmente apresentam melhor mobilidade funcional, habilidade de levantar e sentar, menos medo de cair, melhor estilo de vida e possuem também melhor qualidade de vida em alguns domínios.

Ambos os grupos eram similares em relação às características sociodemográficas. $\mathrm{Em}$ relação ao $\mathrm{IMC}$, chama atenção o fato as médias de ambos indicarem excesso de peso. Curiosamente, a média de IMC dos participantes do GCam se apresenta na faixa de obesidade. Uma provável explicação

Tabela 1. Características dos grupos caminhada e sedentário

\begin{tabular}{lccc} 
& Grupo Caminhada & Grupo Sedentário & $p$ \\
\hline $\mathrm{n}$ & 25 & 26 & 0,14 \\
Mulheres/Homens & $19 / 6$ & $14 / 12$ & 0,79 \\
Idade (anos) & $69,4 \pm 7,3$ & $70,8 \pm 6,8$ & 0,97 \\
Peso $(\mathrm{kg})$ & $72,1 \pm 10,7$ & $68,1 \pm 10,9$ & 0,29 \\
\hline Estatura (cm) & $154,2 \pm 10,5$ & $159,7 \pm 9,1$ & 0,49 \\
\hline IMC $\left(\mathrm{Kg} / \mathrm{m}^{2}\right)$ & $30,4 \pm 3,9$ & $26,7 \pm 3,8$ & \\
\hline
\end{tabular}

IMC: índice de massa corporal; $\mathrm{kg}$ : quilogramas; $\mathrm{cm}$ : centímetro, $\mathrm{Kg} / \mathrm{m}^{2}$ : quilograma por metro quadrado

Tabela 2. Comparação dos resultados dos testes clínicos e estilo de vida entre os grupos

\begin{tabular}{lccc} 
& Grupo Caminhada & Grupo Sedentário & $p$ \\
\hline Timed Up and Go (s) & $9,4 \pm 2,7$ & $14,9 \pm 6,5$ & 0,001 \\
FES-I & $23,2 \pm 5,3$ & $29,0 \pm 10,2$ & 0,014 \\
Teste levanta e senta (s) & $11,2 \pm 5,3$ & $15,3 \pm 9,3$ & 0,06 \\
Estilo de Vida & $79,8 \pm 5,5$ & $67,2 \pm 10,3$ & 0,0001 \\
\hline
\end{tabular}

FES-I: Escala Internacional de Eficácia de Quedas; s: segundos para este fato seria que estes indivíduos tenham aderido ao programa de caminhada justamente devido à obesidade.

A prática regular de atividade física pode prevenir agravos importantes como quedas, devido aos estímulos musculoesqueléticos e somatossensoriais que contribuem para que o indivíduo adquira melhores condições de equilíbrio corporal. ${ }^{27}$ Por outro lado, o sedentarismo associado à senescência pode contribuir para a redução das capacidades físicas envolvidas no controle do equilíbrio e, portanto, predispor os indivíduos idosos a quedas. ${ }^{27,28} \mathrm{Um}$ exemplo desta questão é um estudo recente que, ao comparar o equilíbrio de idosos da comunidade com diferentes níveis de atividade física, verificou que aqueles muito ativos possuíam melhor equilíbrio em relação aos insuficientemente ativos. ${ }^{29}$

Em relação ao desempenho nos testes clínicos, em todos eles os participantes do GCam obtiveram melhores resultados. O resultado de mobilidade funcional e equilíbrio dinâmico (avaliados pelo Timed Up and Go) do GCam, corrobora com uma metanálise que constatou valor de 9,4 segundos para a realização deste teste por indivíduos idosos. ${ }^{30}$ Por outro lado, a média do tempo de execução do GSed é condizente com o verificado em indivíduos propensos a quedas, ${ }^{31}$ uma vez que a realização do TUG em tempo superior a 14 segundos é um indicativo desta propensão.

Embora o TUG seja amplamente utilizado na literatura nacional e internacional, provavelmente por ser de fácil execução e consumir pouco tempo, ${ }^{32}$ ele possui uma limitada habilidade de prever quedas em idosos da comunidade, e por esta razão não deve ser isoladamente utilizado para este fim. ${ }^{33}$ Por esta razão, no presente estudo também foram empregados outros testes clínicos como o teste de levantar e sentar, que está relacionado com a força de membros inferiores e com o equilíbrio. Ao verificar o tempo gasto no teste, observa-se que, em média, o tempo gasto pelos indivíduos do GSed excede 13,6 segundos, o que, segundo Guralnik et al. ${ }^{21}$ indica aumento da incapacidade e morbidade. Já para Buatois et al. ${ }^{22}$ o ponto de corte no tempo de realização do teste seria de 15 segundos, e poderia prever propensão a quedas. A média de tempo gasto para a realização do teste de levantar e sentar no GSed foi muito próxima deste valor $(15,3 \mathrm{~s})$.

$\mathrm{Na}$ faixa etária de 60 a 69 anos, que compreende a maioria dos participantes deste estudo, espera-se que o tempo de execução do teste de levantar e sentar seja por volta de $11,4 s^{23}$ Os participantes do GCam realizaram 
Tabela 3. Comparação dos resultados de qualidade de vida entre os grupos

\begin{tabular}{lccc} 
& Grupo Caminhada & Grupo Sedentário & $\mathrm{p}$ \\
\hline SF-36 (total) & $59,0 \pm 20,1$ & $51,5 \pm 24,8$ & 0,35 \\
\hline Capacidade Funcional & $75,4 \pm 19,9$ & $57,3 \pm 29,2$ & 0,013 \\
\hline Limitações por aspectos físicos & $75,0 \pm 29,7$ & $49,1 \pm 43,1$ & 0,017 \\
Dor & $25,6 \pm 22,0$ & $38,4 \pm 25,1$ & 0,05 \\
\hline Estado Geral de Saúde & $51,2 \pm 12,5$ & $57,1 \pm 13,8$ & 0,116 \\
Vitalidade & $50,8 \pm 11,9$ & $55,8 \pm 13,5$ & 0,166 \\
Aspectos Sociais & $51,1 \pm 13,5$ & $45,9 \pm 12,7$ & 0,164 \\
Limitação por aspectos emocionais & $78,6 \pm 34,5$ & $57,6 \pm 40,6$ & 0,05 \\
Saúde Mental & $56,8 \pm 8,7$ & $56,6 \pm 9,4$ & 0,94 \\
\hline
\end{tabular}

o teste em tempo menor (11,1s em média), ao passo que os voluntários do GSed precisaram de 15,3s, valor superior ao esperado para indivíduos octogenários $(14,8 \mathrm{~s}) .{ }^{23}$ Desta forma, os resultados do presente estudo indicam que idosos praticantes de caminhada apresentamse dentro dos padrões normalidade no que diz respeito ao tempo de realização do teste de levantar e sentar, o que provavelmente tenha colaborado também para o melhor tempo de execução do TUG.

O medo de cair caracteriza-se pela ansiedade ao caminhar ou preocupação excessiva em cair, e pode estar relacionado a fatores como depressão, sentimentos de desamparo, isolamento social e alterações que limitam a mobilidade funcional. ${ }^{34}$ Desta forma, é razoável compreender que aqueles que praticam atividade física regularmente possuam menor medo de cair em relação aos sedentários, pois a prática da caminhada pode melhorar o equilíbrio dinâmico e alguns aspectos emocionais associados ao medo de cair. ${ }^{9,35}$

Ainda em relação ao medo de cair, a pontuação média obtida pelo GCam no instrumento FES-I foi de 23,2, o que indica histórico de queda esporádica. ${ }^{24} \mathrm{~A}$ pontuação mais alta observada no GSed $(29,0)$ se enquadra nesta mesma classificação, ${ }^{24}$ no entanto, este último grupo está a dois pontos de passar a ser classificado como associação a queda recorrente.

Em relação ao estilo de vida, verificou-se diferença significante entre os grupos, sendo esta variável melhor no GCam. A quantificação do estilo de vida é uma tarefa desafiadora porém imprecisa, pois ele é composto por muitas dimensões, naturalmente difíceis de serem constatadas objetivamente. O questionário empregado no presente estudo, estilo de vida FANTASTICO não foi originalmente desenvolvido para indivíduos idosos, porém estudo recente $^{36}$ que o utilizou em idosas constatou que a análise de consistência interna do instrumento revelou um alfa de Cronbach de 0,77, indicando aceitável confiabilidade para esta população.

O estilo de vida dos participantes do GCam foi considerado muito bom, e os indivíduos do GSed tiveram seu estilo de vida avaliado como bom. ${ }^{26}$ É possível que os praticantes de caminhada sejam pessoas que se preocupam com hábitos relacionados à saúde, o que explicaria a adesão ao programa de caminhada, e que este fato, por sua vez, tenha desencadeado outros hábitos saudáveis nestes idosos. Sabe-se que a adoção de um estilo de vida saudável que incluía a prática de uma atividade física regular é extremamente benéfica, pois é uma forma de prevenir ou controlar as doenças e agravos não transmissíveis. ${ }^{17,18}$

Em relação à qualidade de vida, os resultados do presente estudo mostram diferenças significantes entre os GCam e GSed nos domínios capacidade funcional, limitação por aspecto físico, dor e limitação por aspectos emocionais. Apesar disso, não foram observadas diferenças entre os grupos nos domínios estado geral de saúde, vitalidade, aspectos sociais e saúde mental.

O domínio capacidade funcional se constitui num alvo de investimento importante na avaliação e na promoção da saúde de indivíduos idosos. É um aspecto central da qualidade de vida do idoso, que sofre influências com o aumento da idade. No presente estudo, observou-se um bom resultado em termos da capacidade funcional dos idosos participantes do GCam. Este domínio representa a capacidade do indivíduo em desempenhar as atividades da vida diária, as atividades de cuidados pessoais básicos como vestir-se, banhar-se, levantar-se da cama e sentar-se, utilizar o banheiro, comer e caminhar pequenas distâncias, mantendo assim a sua autonomia.
O estudo realizado por Jesus e Silva ${ }^{37}$ constatou que idosos praticantes de atividades físicas tinham boa qualidade de vida, atingindo uma pontuação média de 77,33 \pm 41,25 neste domínio, resultado semelhante observado neste estudo para o GCam. A limitação por aspectos físicos foi o segundo melhor domínio avaliado, obtendo resultado significantemente melhor para os idosos do GCam em relação ao GSed, o que reitera a atividade física como importante fator para a manutenção da autonomia na terceira idade.

No que se refere ao domínio dor, curiosamente, o resultado obtido do GCam foi inferior ao do GSed. Sabe-se que a dor crônica (como doença e não sintoma) pode prejudicar a qualidade de vida dos idosos, ${ }^{38}$ o que pode ter sido observado neste estudo. É possível que, por apresentarem dores diversas, os idosos tenham procurado a caminhada como atividade física e forma de tratamento. Ainda, outra possível explicação seria que a prática regular desta atividade física não esteja sendo realizada com as precauções devidas, como uso de calçados adequados, preparo prévio com aquecimento, dentre outros fatores que poderiam desencadear dores nesses idosos.

Um estudo de revisão ${ }^{39}$ aponta que há evidência limitada a respeito dos benefícios da prática de atividade física na qualidade de vida de idosos da comunidade, e acrescenta que embora a caminhada seja realizada frequentemente pelos idosos, os autores não encontraram outros estudos que avaliassem os efeitos desta atividade sobre a qualidade de vida.

Este estudo possui algumas limitações. Os instrumentos de avaliação utilizados foram medidas indiretas, no entanto, todos testes já haviam sido validados e são corriqueiramente utilizados em estudos nacionais e na prática clínica. A natureza transversal do estudo também não permite estabelecer relações de causa e efeito entre as variáveis estudadas. Por outro lado, os resultados encontrados foram claramente distintos entre os grupos, levando a crer que a participação em grupos de caminhada possa ser benéfica para a melhora da mobilidade funcional, equilíbrio, força e diminuição do medo de cair, bem como para alguns aspectos da qualidade de vida.

Sabe-se que a promoção e a manutenção de um bom nível de atividade física em idosos é imperativo quando se deseja obter um envelhecimento saudável..$^{40}$ Desta forma, a caminhada parece ser uma boa alternativa, no entanto, ensaios clínicos randomizados controlados são desejáveis para esclarecer os efeitos da prática da caminhada na dor e em outros aspectos relevantes na terceira idade. 


\section{CONCLUSÃO}

Em conclusão, idosos participantes regulares de caminhada possuem melhor mobilidade funcional, melhor habilidade de levantar e sentar, melhor estilo de vida, menos medo de cair e melhores resultados nos domínios de capacidade funcional, limitações por aspectos físicos e limitações por aspectos emocionais do que indivíduos idosos sedentários.

\section{REFERÊNCIAS}

1. Miranda GMD, Mendes ACG, Silva ALA. O envelhecimento populacional brasileiro: desafios e consequências sociais atuais e futuras. Rev Bras Geriatr Gerontol. 2016;19(3):507-19. DOI: https:// doi.org/10.1590/1809-98232016019.150140

2. Veras R. Envelhecimento populacional contemporâneo: demanda, desafios e inovações. Rev Saúde Pública. 2009;3(43):548-54. DOI: https:// doi.org/10.1590/S0034-89102009000300020

3. Alfieri FM, Moraes MCL. Envelhecimento e o controle postural. Saúde Col. 2008; 4(19):30-3.

4. Alfieri FM, Silva CR, Alcântara CC, Santos KIS, Melo FC. Equilíbrio e mobilidade funcional em indivíduos independentes para o autocuidado de diferentes faixas etárias. Rev Kairós. 2016;18(4):151-63.

5. Brovold T, Skelton DA, Bergland A. Older adults recently discharged from the hospital: effect of aerobic interval exercise on health-related quality of life, physical fitness, and physical activity. J Am Geriatr Soc. 2013;61(9):1580-5. DOI: https://doi. org/10.1111/jgs.12400

6. Moore SC, Patel AV, Matthews $\mathrm{CE}$, Berrington de Gonzalez A, Park Y, Katki HA, et al. Leisure time physical activity of moderate to vigorous intensity and mortality: a large pooled cohort analysis. PLoS Med. 2012;9(11):e1001335. DOI: https://doi. org/10.1371/journal.pmed.1001335

7. Warburton DER, Nicol CW, Bredin SSD. Health benefits of physical activity: the evidence. CMAJ. 2006;174(6):801-9. DOI: https://doi.org/10.1503/ cmaj.051351

8. Kemmler W, von Stengel S. Exercise frequency, health risk factors, and diseases of the elderly. Arch Phys Med Rehabil. 2013;94(11):2046-53. DOI: https://doi. org/10.1016/j.apmr.2013.05.013

9. Hatta A, Nishihira $Y$, Higashiura T. Effects of a single bout of walking on psychophysiologic responses and execute function in elderly adults: a pilot study. Clin Interv Aging. 2013;8:945-52. DOI: https://doi. org/10.2147/CIA.S46405

10. Lee YJ, Hung WL. The relationship between exercise participation and well-being of the retired elderly. Aging Ment Health. 2011;15(7):873-81. DOI: https:// doi.org/10.1080/13607863.2011.569486

11. Mazzeo RS, Cavanagh P, Evans WJ, Fiatarone M, Hagberg J, McAuley E, et al. Exercise and physical activity for older adults. Med Sci Sports Exerc. 1998;30: 992-1008.
12. Falck RS, Davis JC, Milosevic E, Liu-Ambrose T. How much will older adults exercise? A feasibility study of aerobic training combined with resistance training. Pilot Feasibility Stud. 2017;3:2. DOI: https://doi. org/10.1186/s40814-016-0116-5

13. Queiroz BM, Coqueiro RS, Leal Neto JS, Borgatto AF, Basrbosa AR, Fernandes $\mathrm{MH}$. Physical inactivity among non-institutionalized elderly individuals: a population-based study. Cien Saude Colet. 2014;19(8):3489-96. DOI: https://doi. org/10.1590/1413-81232014198.19882013

14. Killingback C, Tsofliou F, Clark C. Older people's adherence to community-based group exercise programmes: a multiple-case study. BMC Public Health. 2017;25;17(1):115. DOI: https://doi. org/10.1186/s12889-017-4049-6

15. Pousas FM, Rodrigues AS, Duarte IA, Bispo AS. Estudo comparativo das alterações na aptidão cardiovascular em idosos praticantes e não participantes de caminhada em Alvarenga, MG. Mundo Saúde. 2007;31(4):489-93.

16. Davison RCR, Grant $\mathrm{S}$. Is walking sufficient exercise for health? Sports Med. 1993;16:369-73.

17. Murtagh EM, Murphy $\mathrm{MH}$, Boone-Heinonen J. Walking: the first steps in cardiovascular disease prevention. Curr Opin Cardiol. 2010;25(5):490-6. DOI https://doi.org/10.1097/HCO.0b013e32833ce972

18. Morris JN, Hardman AE. Walking to health. Sports Med. 1997;23(5):306-32. DOI: https://doi. org/10.2165/00007256-199723050-00004

19. Chapman RS, Syrjala KL. Measurement of pain. In: Bonica JJ. The management of pain. Londres: Lea \& Febiger; 1990. p. 580-94.

20. Podsiadlo D, Richardson S. The timed "Up \& Go": a test of basic functional mobility for frail elderly persons. J Am Geriatr Soc. 1991;39(2):142-8. DOI: $\quad$ https://doi.org/10.1111/j.1532-5415.1991. tb01616.x

21. Guralnik JM, Ferrucci L, Pieper CF, Leveille SG, Markides KS, Ostir GV, et al. Lower extremity function and subsequente disability: consistency across studies, predictive models, and value of gait speed alone compared with the short physical performance battery. J Gerontol A Biol Sci Med Sci. 2000;55(4):22131. DOI: https://doi.org/10.1093/gerona/55.4.M221

22. Buatois S, Miljkovic D, Manckoundia P, Gueguen R, Miget $P$, Vançon $G$, et al. Five times sit to stand test is a predictor of recurrent falls in healthy communityliving subjects aged 65 and older. J Am Geriatr Soc 2008;56(8):1575-7. DOI: https://doi.org/10.1111/ j.1532-5415.2008.01777.x

23. Bohannon RW. Reference values for the fiverepetition sit-to-stand test: a descriptive metaanalysis of data from elders. Percept Mot Skills. 2006;103(1): 215-22. DOI: https://doi.org/10.2466/ pms.103.1.215-222

24. Camargos FFO, Dias RC, Dias JMD, Freire MTF. Adaptação transcultural e avaliação das propriedades psicométricas da Falls Efficacy Scale - International em idosos Brasileiros (FES-I-BRASIL). Rev Bras Fisioter 2010;14(3):237-43. DOI: https://doi.org/10.1590/ S1413-35552010000300010

25. Ciconelli RM, Ferraz MB, Santos WS, Meinão I, Quaresma MR. Tradução para a língua portuguesa e validação do questionário genérico de qualidade de vida SF-36 (Brasil SF-36). Rev Bras Reumatol. 1999;39(3):143-50.
26. Anẽz CRR, Reis RS, Petroski EL. Versão brasileira do questionário "Estilo de Vida Fantástico": tradução e validação para adultos jovens. Arq Bras Cardiol. 2008;2(91):102-09.

27. Skelton DA. Effects of physical activity on postural stability. Age Ageing. 2001;30(4):33-9. DOI: https:// doi.org/10.1093/ageing/30.suppl_4.33

28. Silva EC, Duarte NB, Arantes PMM. Estudo da relação entre o nível de atividade física e o risco de quedas em idosas. Fisioter Pesq. 2011;18(1):23-30. DOI: https:// doi.org/10.1590/S1809-29502011000100005

29. Dias RA, Borba W, Martins MS, Alfieri FM. Nível de atividade física e equilíbrio em idosos da comunidade. Lecturas Ed Física y Dep. 2014; 19(194):1.

30. Bohannon RW. Reference values for the timed up and go test: a descriptive meta-analysis. J Geriatr Phys Ther. 2006;29(2):64-8. DOI: https://doi. org/10.1519/00139143-200608000-00004

31. Shumway-Cook A, Brauer S, Woollacott M. Predicting the probability for falls in community-dwelling older adults using the Timed Up \& Go Test. Phys Ther. 2000;80(9):896-903.

32. Alfieri FM, Riberto M, Gatz LS, Ribeiro CPC, Battistella LR. Uso de testes clínicos para verificação do controle postural em idosos saudáveis submetidos a programas de exercícios físicos. Acta Fisiatr. 2010;17(4):153-8.

33. Barry E, Galvin R, Keogh C, Horgan F, Fahey T. Is the Timed Up and Go test a useful predictor of risk of falls in community dwelling older adults: a systematic review and meta-analysis. BMC Geriatr. 2014;1(14):14. DOI: https://doi.org/10.1186/14712318-14-14

34. Antes DL, Schneider IJC, Benedetti TRB, D'orsi E. Medo de queda recorrente e fatores associados em idosos de Florianópolis, Santa Catarina, Brasil. Cad Saúde Pública. 2013;29(4):758-68. DOI: https://doi. org/10.1590/S0102-311X2013000800013

35. Okubo $Y$, Osuka $Y$, Jung $S$, Rafael F, Tsuimoto T, Aiba $\mathrm{T}$, et al. Walking can be more effective than balance training in fall prevention among community-dwelling older adults. Geriatr Gerontol Int. 2016;16(1):118-25. DOI: https://doi.org/10.1111/ggi.12444

36. Lima ARS, Portes LA, Oliveira NC, Alfieri FM. Limiar de tolerância de dor à pressão, estilo de vida, força muscular e capacidade funcional em idosas com sarcopenia. Acta Fisiatr. 2016;23(2):73-7.

37. Jesus DF, Silva AF. Percepção da qualidade de vida por idosos praticantes e não praticantes de exercícios resistidos: análise do projeto vida corrida. Lecturas Ed Física y Dep. 2010;15(149):1.

38. Cunha LL, Mayrink WC. Influência da dor crônica na qualidade de vida em idosos. Rev Dor. 2011;12(2):120-4. DOI: https://doi.org/10.1590/ S1806-00132011000200008

39. Oliveira AC, Oliveira NMD, Arantes PMM, Alencar MA. Qualidade de vida em idosos que praticam atividade física - uma revisão sistemática. Rev Bras Geriatr Gerontol. 2010;13(2):301-12. DOI: https:// doi.org/10.1590/S1809-98232010000200014

40. Olanrewaju O, Kelly S, Cowan A, Brayne C, Lafortune L. Physical activity in community dwelling older people: a systematic review of reviews of interventions and context. PLoS One. 2016;11(12):e0168614. DOI: https://doi.org/10.1371/journal.pone.0168614 